This document is the accepted manuscript version of the following article:

Seibe1, J., Parschau, M., \& Ernst, K. H. (2020). Double layer crystallization of heptahelicene on noble metal surfaces. Chirality, 32(7), 975-980.

https://doi .org/10.1002/chir.23235

\title{
Double layer crystallization of heptahelicene on noble metal surfaces
}

\author{
Johannes Seibel[a], Manfred Parschau ${ }^{[a]}$, Karl-Heinz Ernst ${ }^{*[a, b, c]}$
}

Keywords: chiral crystallization, heptahelicene, scanning tunneling microscopy, surface science

Abstract: Resolution of enantiomers of chiral compounds via crystallization is the dominant method in chemical industry, but chiral recognition at the molecular level during this process is still poorly understood. Using single metal surfaces in ultrahigh vacuum as model system, the enantio-related transition from the monolayer structure into a double layer of the racemic mix- ture of heptahelicene has been studied with scanning tunnelling microscopy. Submolecular resolution reveals enantiopure second layers on $\mathrm{Ag}(111)$ and almost enantiopure second layers on $\mathrm{Au}(111)$. In analogy to previous results on $\mathrm{Cu}(111)$ it is concluded that transition from the 2D first layer racemate into a layered racemate occurs.

\section{Introduction}

As heterogeneous nucleation at surfaces requires a much lower supersaturation than homogeneous nucleation in solution, the surface structure is significantly involved in stereochemical recognition during crystallization. In that respect, the problem of chiral recognition, notably whether a compound undergoes resolution into a conglomerate or aggregates into a racemic crystal, could be largely influenced by the structure of surface. The problem of conglomerate versus racemate crystallization - even more than 170 years after Pasteur's seminal discovery of the conglomerate crystallization of ammonium sodium tartrate ${ }^{1}$ - is still not understood today. An early approach of explanation was based on crystal densities, ${ }^{2,3}$ but a more recent evaluation revealed a strong bias. ${ }^{4}$ The problem calls for studies of appropriate model systems in order to better understand chiral recognition in crystallization.5,6 One approach therefore is studying the aggregation of chiral molecules on single-crystalline metal surfaces in ultrahigh vacuum. ${ }^{7,8}$

The on-surface crystallization of helical aromatic hydrocarbons, so-called helicenes, serves as such a model system. ${ }^{9}$ Beyond chiral recognition, however, helicenes on surfaces may also show interesting new phenomena, such as electron spin selectivity, electro-mechanics or selective chiroptical response. ${ }^{10-13}$

There have been numerous reports on self-assembly and crystallization of helicenes on different surfaces studied with scanning tunneling microscopy (STM). On $\mathrm{Cu}(100)$, for example, racemic heptahelicene $\left(\mathrm{rac}-[7] \mathrm{H}, \mathrm{C}_{30} \mathrm{H}_{18}\right.$, Fig. 1) crystallized into a two-dimensional (2D) conglomerate. At coverages up to one monolayer enantiopure quadruplets were observed. ${ }^{14}$ Interestingly, the same motif was identified for 5-amino[6]helicene on the same surface,,$^{15}$ but by alloying with tin racemate formation was induced. ${ }^{16} \mathrm{~A}$ conglomerate-to-racemate transition was also observed on $\mathrm{Ag}(100)$ for [7] $\mathrm{H}$. Enantiopure quadruplets at small coverage turned into racemic zigzag rows with increasing coverage. ${ }^{17}$ Moreover, cyano-functionalized $[7] \mathrm{H}$ forms a $2 \mathrm{D}$ conglomerate on $\mathrm{Cu}(111),{ }^{18}$ while bromo, benzo and $\mathrm{S}$ acetylthiolate functionalized $[7] \mathrm{H}$ formed zigzag racemate structures ${ }^{19}$ Finally, it seems noteworthy that the self-assembly of 9,9'-bis[7]H on copper and gold surfaces lead to diastereoselective separation into a racemate phase plus domains build up only by $(M, P)$-isomers. ${ }^{20,21}$

Most relevant here, however, is the previously reported $2 \mathrm{D}$ aggregation of [7] $\mathrm{H}$ on the three (111) surfaces of $\mathrm{Cu}, \mathrm{Ag}$ and $\mathrm{Au}^{22,23}$ In all three cases zigzag rows with alternating enantiomers were observed for the racemate at coverages up to one monolayer. At coverages beyond a monolayer transition into a racemate with enantiopure layers was observed on $\mathrm{Cu}(111)$, in which small enantiomeric excess caused enantiospecific separation into separate layers. ${ }^{24}$ Multilayers of enantiopure [7] $\mathrm{H}$ showed a transmission of handedness from the molecular level into enantiomorphous Moiré patterns. ${ }^{25} \mathrm{~A}$ transition from a racemate phase to a conglomerate phase in the first layer of pentahelicene was found to be initiated once the coverage exceeded that of a saturated monolayer. ${ }^{26}$

Here, the second layer-crystallization of racemic [7] $\mathrm{H}$ on the surfaces of $A g(111)$ and $A u(111)$, studied by means of scanning tunneling microscopy (STM), is presented. For both systems the transition from a $2 \mathrm{D}$ racemate to a layered racemate with enantiopure layers has been observed. On the $\mathrm{Ag}(111)$ surface, lattice vectors of the [7] $\mathrm{H}$ domains are aligned parallel to highsymmetry directions of the metal surface lattice, while on $\mathrm{Au}(111)$ oblique angles between the [7] $\mathrm{H}$ lattice and the substrate metal lattice are obtained, leading to enantiomorphism in form of mirror domains.

\section{Materials and Methods}

The experiments have been carried out in an ultrahigh vacuum (UHV) system (base pressure $<5 \cdot 10^{-10} \mathrm{mbar}$ ) equipped with an variable-temperature scanning tunneling microscope (Omicron Nanotechnology). The (111) crystals were prepared by cycles of $\mathrm{Ar}^{+}$ion bombardment and subsequent annealing to $800 \mathrm{~K}$. [7] $\mathrm{H}$

[a] Dr. J. Seibel, Dr. M. Parschau, Prof. Dr. K.-H. Ernst Empa, Swiss Federal Laboratories for Materials Science and Technology, Überlandstrasse 129 CH-8600 Dübendorf, Switzerland E-mail: Karl-Heinz.Ernst@empa.ch

[b] Prof. Dr. K.-H. Ernst Department of Chemistry University of Zurich, $\mathrm{CH}-8057$ Zürich, Switzerland

[c] Prof. Dr. K.-H. Ernst Nanosurf Lab Institute of Physics of the Czech Academy of Sciences Cukrovarnická 10 Prague 6 CZ 16200 Czech Republic

Received: ((will be filled in by the editorial staff)) Revised: ((will be filled in by the editorial staff)) Published online: ((will be filled in by the editorial staff)) 

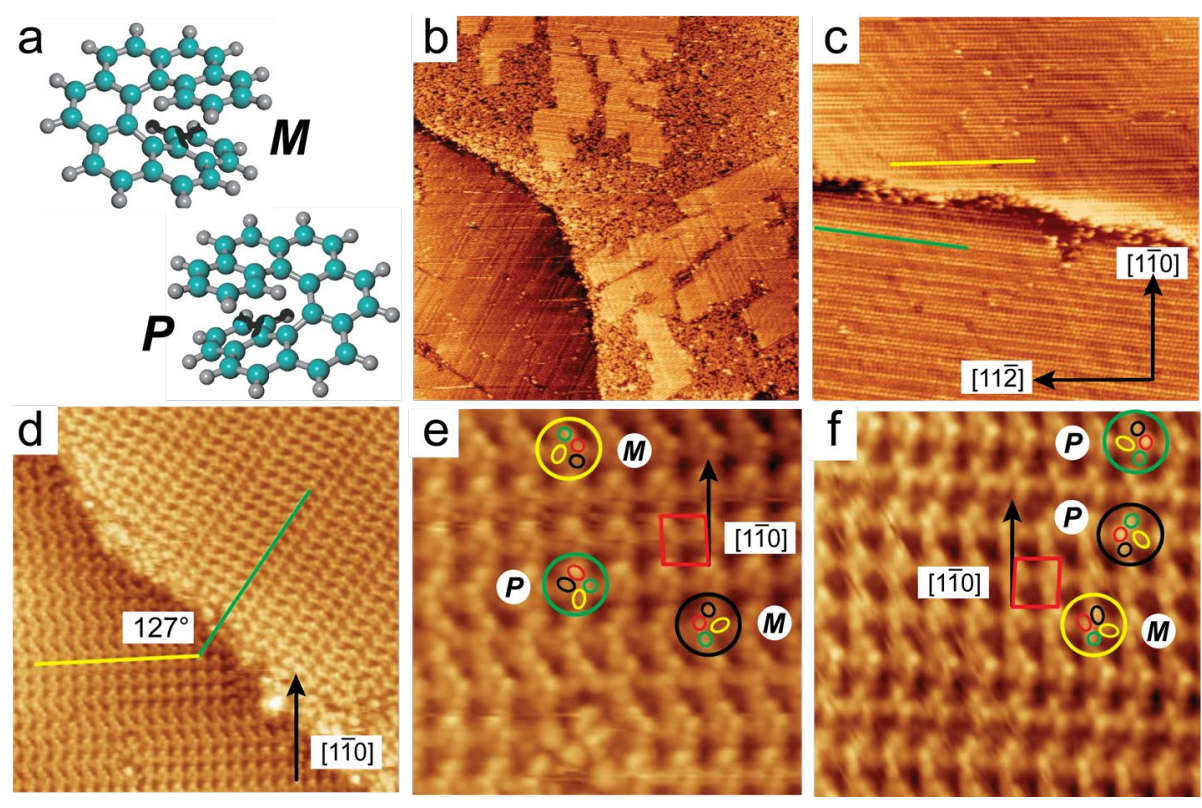

Figure 1. STM images of second layer growth of [7]H on Au(111). (A) Ball-and-stick molecular models of [7]H enantiomers. (B) Long-range STM image $(200 \mathrm{~nm} \times 200 \mathrm{~nm}, 2.56 \mathrm{~V}, 10 \mathrm{pA})$ of a partially completed second layer, showing ordered double layer islands and disordered $1^{\text {st }}$ layer areas. (C) STM image $(100 \mathrm{~nm} \times 100 \mathrm{~nm}, 2.73 \mathrm{~V}, 10 \mathrm{pA})$ of the saturated second layer, showing dark and bright stripes running parallel with the molecular lattice vectors, as indicated by the green and yellow lines. These are aligned at opposite oblique angles with respect to the Au surface [11̄2] direction. The herringbone reconstruction of the $\mathrm{Au}(111)$ surface is still observed underneath the molecular double layer of rac-[7]H in the STM images. (D) STM image $(30 \mathrm{~nm} \times 30 \mathrm{~nm}, 2.78 \mathrm{~V}, 10 \mathrm{pA})$ showing a grain boundary between two double layer domains. The boundary coincides with a monoatomic step between two terraces of the $\mathrm{Au}(111)$ surface. The two domains are relatively aligned to each other in an angle of $127^{\circ}$. (E,F) High-resolution STM images $(10 \mathrm{~nm} \times 10 \mathrm{~nm}, 2.78 \mathrm{~V}, 10 \mathrm{pA}$ ) of enantiomorphous domains. The unit cells (red tetragons) are tilted in opposite way with respect to the close-packed [110] surface direction (black arrows). A single molecule appears as four lobes, running from bright to dark (red-green-yellow-black) counterclockwise $(M-[7] \mathrm{H})$ or clockwise $(P-[7] \mathrm{H})$. The majority of the molecules shown in $(E)$ consists of $M$ $[7] \mathrm{H}$ and the majority in $(\mathrm{F})$ of $P-[7] \mathrm{H}$. In part, entire rows with molecules rotated by $180^{\circ}$ in the second layer (black circles) as well as molecules of opposite helicity are identified.

(Chiracon $\mathrm{GmbH}$, Luckenwalde) was deposited by thermal sublimation from an effusion cell $\left(160^{\circ} \mathrm{C}\right)$ onto the surface held at room temperature. STM images in constant current mode were acquired after cooling the sample to $60 \mathrm{~K}$ with liquid helium.

\section{Results and Discussion}

\section{DOUBLE LAYER GROWTH ON AU(111)}

On $\mathrm{Au}(111)[7] \mathrm{H}$ grows into $(M) /(P)$ zigzag rows, aligned parallel to the three high-symmetry directions of the surface. ${ }^{23}$ Once the coverage on the surface exceeds the amount of molecules that can be accommodated into the first molecular layer, double layer crystallization occurs. At coverages between monolayer coverage and the amount needed for completion of the second layer, a depleted disordered first layer coexists with ordered double-layer islands (Figure 1b). The completed double layer shows bright stripes within the domains which are oriented in oblique angles with respect to the high-symmetry directions of the metal substrate lattice (Figure 1c). Hence, an arrangement in form of mirror domains is established, which means that certain domains cannot be brought into superposition by translation and rotation only but would require reflection at mirror planes perpendicular to the surface. The surface structure of the gold surface is not affected, as the well-known herringbone surface reconstruction is still observed through the molecular double-layer (Figure 1c). Mirror domain boundaries typically coincide with monoatomic steps of the surface, which means that atomically flat terraces are usually covered by a single domain. The angle spanned between mirror domains is $7^{\circ}$ and that of rotational domains $120^{\circ}$ (Figure1d).

STM images at larger magnification reveal submolecular contrast in which single molecules appear as four-lobe patterns. From previous studies performed with $(M)$-[7]H double-layers on $\mathrm{Cu}(111)$ the absolute handedness of the molecules can be determined from this pattern. Running from brighter to darker in counterclockwise manner identifies the $(M)$-enantiomer. In Figures $1 \mathrm{e}$ and $1 \mathrm{f}$ the four-lobe pattern has been highlighted by colored ellipses. A clockwise sequence of red-green-yellowblack marks $(P)$-enantiomers, while the counterclockwise redgreen-yellow-black sequence stands for $(M)$-enantiomers. Interestingly, the second layers are not entirely enantiopure but accommodate the other enantiomer in form of rows, which explains the different contrast in form of the aforementioned bright stripes. In addition, entire rows may have the majority enantiomer rotated by 180 degrees. The majority of the molecules in the domain shown in Figure 1e are $(M)-[7] \mathrm{H}$, while the domain shown in Figure $1 \mathrm{f}$ contains mainly $(P)-7 \mathrm{H}$.

The unit cells, as indicated by red tetragons, are almost quadratic. The molecular lattice tilt with respect to the [110] direction of the gold(111) surface (black arrows) is small $\left( \pm 3.5^{\circ}\right.$, Figs. 1e,f)

\section{DOUBLE LAYER GROWTH ON Ag(111)}

Figure 2 shows STM images of the growth of rac-[7] $\mathrm{H}$ on the (111) surface of silver. Once the coverage of rac-[7] $\mathrm{H}$ on $\mathrm{Ag}(111)$ is increased above the monolayer coverage, second layer islands from upon cooling, while the order in the first layer disappears. At a coverage between the saturated monolayer 
and saturated second layer, the second layer islands are embedded in disordered areas and bright stripes are observed again in the second layer (Fig. 2a). However, in contrast to [7] $\mathrm{H}$ on $\mathrm{Au}(111)$ these stripes disappear in the saturated second layer and well-ordered domains extending over entire terraces are formed (Fig. 2b).
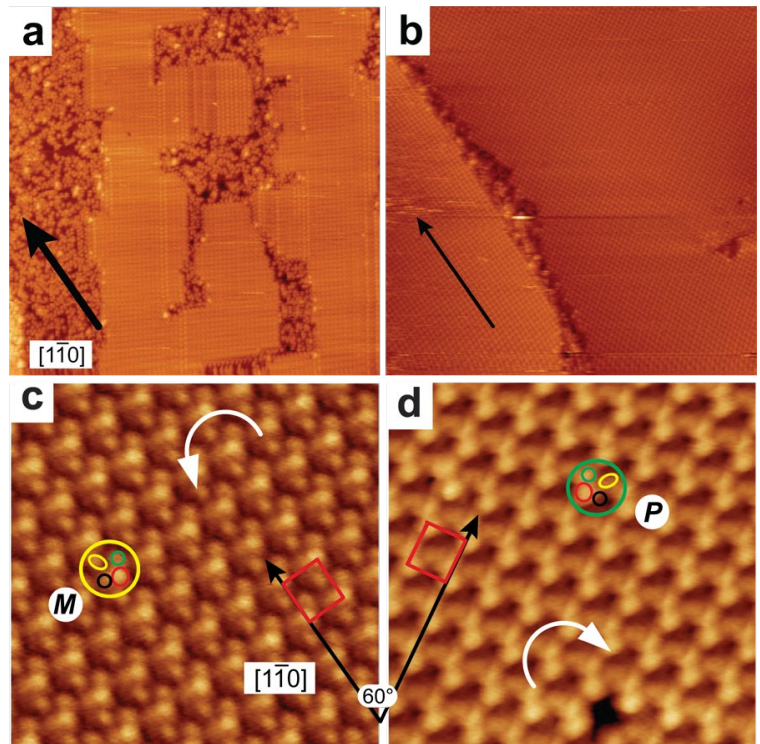

Figure 2. STM images of rac-[7] $\mathrm{H}$ on $\mathrm{Ag}(111)$ showing second layer growth. (A) Formation of ordered second layer islands while the first layer at local lower density stays disordered. The [110] direction in the $\mathrm{Ag}(111)$ plane is indicated by an arrow $(100 \mathrm{~nm} \times 100 \mathrm{~nm},-2.84$ $\mathrm{V}, 28 \mathrm{pA}$ ). (B) Closed-packed double layer of rac-[7] H on $\mathrm{Ag}(111)$. The second layer lattice vector of one domain runs parallel to the [11̄0] direction $(100 \mathrm{~nm} \times 100 \mathrm{~nm},-2.78 \mathrm{~V}, 34 \mathrm{pA})$. (C,D) High resolution STM images $(10 \mathrm{~nm} \times 10 \mathrm{~nm})$ of the second layer lattices of two different domains. A single molecule appears in four hillocks. These are arranged clockwise or counterclockwise, reflecting the absolute handedness of the molecules.

High resolution STM images show that the second layer is enantiopure (Fig. 2c,d). Again, the absolute handedness of the domains can be determined by going from bright to dark contrast within a single molecule as illustrated for two domains of opposite chirality (Fig. 2c,d). In contrast to the double layer on $\mathrm{Au}(111)$, the domains of $\mathrm{rac}-[7] \mathrm{H}$ on $\mathrm{Ag}(111)$ do not show oblique tilt angles. The lattice vectors are aligned parallel to the [ $\left.\begin{array}{lll}1 & \overline{1} & 0\end{array}\right]$ surface direction and therefore, enantiomorphous domains just run into the opposite direction. Together with the threefold symmetry of the surface, which allows rotational domains of $120^{\circ}$, this leads to the observed angle of $60^{\circ}$ (Fig. 2c,d).

Overall, the second layer growth of rac-[7] $\mathrm{H}$ is very similar on all investigated surfaces, that is, second layer islands start to grow as soon as the coverage is increased above the saturated monolayer coverage. The areas between these islands exhibit complete disorder or reduced ordering in the case of $\mathrm{Cu}(111)$.

The mechanism of the observed second layer island growth and disorder in adjacent areas can be explained under the assumption that the critical temperature for nucleation crystallization of the second layer is higher compared to the crystallization temperature of the first layer racemic zigzag row structure. As soon as the coverage exceeds the one of the saturated monolayer during deposition at RT, molecules become located in the second layer. Upon cooling, double layer island nucleation and growth starts before first-layer crystallization. It seems that during second layer growth, molecules are transferred from the first layer into the second layer. As soon as this process ceases with decreasing temperature the first layer structure may start to form. However, due to the transfer of molecules from the first into second layer, the density in the first layer became too low to form ordered structures, resulting in disorder in the remaining areas adjacent to the second layer islands. First layer ordering is only observed for coverages very close to monolayer saturation.

The rac-[7]H first layer zigzag rows on $\mathrm{Au}(111)$ and $\mathrm{Ag}(111)$ were reported to be aligned parallel to the [1 $1 \overline{1} 0]$ surface direction. ${ }^{23}$ Interestingly, enantiomorphism is established due to the double layer crystallization on $\mathrm{Au}(111)$, as the second layer lattice vectors now show an oblique tilt angle with respect to the [110] surface direction. Even though the unit cell vector is parallel to the [1 $1 \overline{1} 0]$ surface direction, the second layer on $\mathrm{Ag}(111)$ also exhibits enantiomorphism because of the opposite handedness of the molecules in the homochiral second layer structure. Hence, the chiral expression of rac-[7] $\mathrm{H}$ on $\mathrm{Ag}(111)$ and $\mathrm{Au}(111)$ changes with the second layer formation.

Due to the change from a racemic structure in the saturated monolayer to an enantiopure second layer structure, the bottom layer structure has likely changed from the racemic zigzag rows in the monolayer. From the presented results here, exact conclusions on the first layer structure are not possible. However, a rough estimate of areas of the two domain types allows basically two possibilities. Either a conglomerate is present, with enantiopure double layer domains, or a racemate, with the other enantiomer in the first layer. Fortunately, the bottom layer structure for $\mathrm{rac}-[7] \mathrm{H}$ on $\mathrm{Cu}(111)$ has been determined previously. ${ }^{24}$ On the one hand, electron diffraction experiments revealed the same periodicity as observed for the top layer in STM. Moreover, by the so-called 'sergeant-andsoldier' experiment, single enantiomorphism was established by using an enantiomeric excess of $\pm 7 \%$. As the consequence only one enantiomer was located in the second layer of the entire sample. Being still close to a ratio of $1: 1$, the racemate crystal scenario was substantiated. Moreover, enantiopure double layers on $\mathrm{Ag}(111)$ and $\mathrm{Au}(111)$ are expected to show, as observed for enantiopure double layers on $\mathrm{Cu}(111)$, Moiré patterns, which is not the case. Because of the striking similarities for all structures observed for monolayers and second layers for all surfaces, it is concluded that the bottom layers in the double layer samples are as well of opposite handedness than the second layer. Enantiomeric excess experiments for the rac-[7] $\mathrm{H} / \mathrm{Ag}(111)$ system with its mirror domains should then show also single mirror domain enantiomorphism. And it would be interesting to see if for the rac-[7] $\mathrm{H} / \mathrm{Ag}(111)$ system, with the absence of mirror domain enantiomorphism, such enantiospecific surface dewetting, i.e., only one enantiomer in the second layer, can be achieved.

Even though the observed structures of the mono- and double layers are very similar on the (111) surfaces of $\mathrm{Ag}$ and $\mathrm{Au}$ there are small differences in the unit cells. On $\mathrm{Ag}(111)$ both enantiomers are adsorbed with the unit cell running along a high-symmetry surface direction (zig-zag rows and double layer), while the unit cell of the double layer has a small oblique angle on $\mathrm{Au}(111)$. This is surprising as the next neighbour distances for both (111) surfaces are $2.88 \AA$. Au(111), however, shows a herringbone reconstruction in the top layer, which might induce a very small strain into the molecular layer if its lattice would follow exactly a high-symmetry direction, as observed here for $\mathrm{Ag}(111)$. The first-layer zigzag rows on $\mathrm{Au}(111)$ 
appeared only in relatively short segments, which supports the strain scenario. ${ }^{23}$

\section{Conclusions}

The double layer crystallization of racemic heptahelicene on the surfaces of $\mathrm{Au}(111)$ and $\mathrm{Ag}(111)$ shows a remarkable transition from the monolayer structures once a small excess of racemate beyond the saturated monolayer coverage is installed. From the racemic monolayer film, in which both enantiomers alternate in zigzag rows aligned along a high-symmetry direction of the substrate, the system turns into a double layer crystal, in part $(\mathrm{Au}(111))$ expressing enantiomorphism in form of mirror domains. Based on remarkable analogies to the rac$[7] \mathrm{H} / \mathrm{Cu}(111)$ system, it is concluded that the double layered crystals consist of enantiopure layers of both enantiomers.

\section{Acknowledgements}

Financial support by the Swiss National Science Foundation is gratefully acknowledged.

\section{REFERENCES AND NOTES}

1. Pasteur, L. Recherches sur les relations qui peuvent exister entre la forme cristalline: la composition chimique et les sens de la polarisation rotatoire. Ann. Phys. 1848;24:442-459.

2. Wallach O. Zur Kenntniss der Terpene und der ätherischen Oele 34. Justus Liebigs Ann. Chem. 1895;286:119-143.

3. Ernst K-H. On the validity of calling Wallach"s Rule Wallach's Rule. Isr. J. Chem. 2016;57:24-30.

4. Brock CP, Schweizer WB, Dunitz JD. On the validity of Wallach's rule: on the density and stability of racemic crystals compared with their chiral counterparts. J. Am. Chem. Soc. 1991;113:9811-9820.

5. Pérez-Garíca L, Amabilino DB. Spontaneous resolution, whence and whither: from enantiomorphic solids to chiral liquid crystals, monolayers and macro- and supramolecular polymers and assemblies. Chem Soc Rev. 2007;36(6):941-967.

6. Pérez-Garíca L, Amabilino DB. Spontaneous resolution under supramolecular control. Chem Soc Rev. 2002;31(6):342-356.

7. Ernst, K.-H. Molecular chirality in surface science. Surf. Sci. 2013;613:1-5.

8. Raval R. Chiral expression from molecular assemblies at metal surfaces: insights from surface science techniques. Chem. Soc. Rev. 2009;38:707-721.

9. Ernst K-H. Stereochemical Recognition of Helicenes on Metal Surfaces. Acc. Chem. Res. 2016;49:1182-1190.

10. Kiran V, Mathew SP, Cohen SR, Hernández Delgado I, Lacour J, Naaman R. Helicenes-a new class of organic spin filter. Adv. Mater. 2016;28:1957-1962.
11. Kettner M, Maslyuk VV, Nürenberg D, Seibel J, Gutierrez R, Cuniberti G, Ernst K-H, Zacharias H. Chirality-dependent electron spin filtering by molecular monolayers of helicenes. $J$. Phys. Chem. Lett. 2018;9:2025-2030.

12. Large converse piezoelectric effect measured on a single molecule on a metallic surface. J. Am. Chem. Soc. 2018;140:940-946.

13. Yang $Y$, da Costa RC, Fuchter MJ, Campbell AJ. Circularly polarized light detection by a chiralorganic semiconductor transistor. Nat. Photonics 2013;7:634-638.

14. Seibel J, Zoppi L, Ernst K-H. 2D conglomerate crystallization of heptahelicene. Chem. Commun. (Camb.) 2014;50:8751-8753.

15. Ascolani H, van der Meijden MW, Cristina LJ, Gayone JE, Kellogg RM, Fuhr JD, Lingenfelder M. Van der Waals interactions in the self-assembly of 5-amino[6]helicene on $\mathrm{Cu}(100)$ and $\mathrm{Au}(111)$. Chem. Commun. (Camb.) 2014;50:13907-13909.

16. Fuhr JD, van der Meijden MW, Cristina LJ, guez LMRX, Kellogg RM, Gayone JE, Ascolani H, Lingenfelder M. Chiral expression of adsorbed (MP) 5-amino[6]helicenes: from random structures to dense racemic crystals by surface alloying. Chem. Commun. (Camb.) 2017;53:130-133.

17. Seibel J, Parschau M, Ernst K-H. From homochiral clusters to racemate crystals: viable nuclei in $2 \mathrm{D}$ chiral crystallization. J. Am. Chem. Soc. 2015;137:7970-7973.

18. Stöhr M, Boz S, Schär M, Nguyen M-T, Pignedoli CA, Passerone D, Schweizer WB, Thilgen C, Jung TA, Diederich $\mathrm{F}$. Self-assembly and two-dimensional spontaneous resolution of cyano-functionalized [7] helicenes on $\mathrm{Cu}(111)$. Angew. Chem. Int. Ed. 2011;50:9982-9986.

19. Mairena A, Mendieta JI, Stetsovych O, Terfort A, Stará IG, Starý I, Jelínek P, Ernst K-H. Heterochiral recognition among functionalized heptahelicenes on noble metal surfaces. Chem. Comm. (Camb.) 2019;55:10595-10598.

20. Mairena A, Parschau M, Seibel J, Wienke M, Rentsch D, Terfort A, Ernst K-H. Diastereoselective self-assembly of bisheptahelicene on $\mathrm{Cu}(111)$. Chem. Commun. (Camb.) 2018;54:8757-8760.

21. Mairena A, Wäckerlin C, Wienke M, Grenader K, Terfort A, Ernst K-H. Diastereoselective Ullmann coupling to bishelicenes by surface topochemistry. J. Am. Chem. Soc. 2018;140:15186-15189.

22. Parschau M, Fasel R, Ernst K-H. Coverage and enantiomeric excess dependent enantiomorphism in twodimensional molecular crystals. Cryst. Growth Des. 2008;8:1890-1896.

23. Seibel J, Parschau M, Ernst K-H. Two-dimensional crystallization of enantiopure and racemic heptahelicene on $\mathrm{Ag}(111)$ and Au(111). J. Phys. Chem. C 2014;118:2913529141.

24. Parschau M, Ernst K-H. Disappearing enantiomorphs: single handedness in racemate crystals. Angew. Chem. Int. Ed. Engl. 2015;54:14422-14426. 
25. Parschau M, Ellerbeck U, Ernst K-H. Chirality transfer by epitaxial mismatch in multi-layered homochiral molecular films. Colloids and Surfaces A: Physicochem. Eng. Aspects 2010;354:240-245.

26. Mairena A, Zoppi L, Seibel J, Tröster AF, Grenader K,

Parschau M, Terfort A, Ernst K-H. Heterochiral to homochiral

transition in pentahelicene $2 \mathrm{D}$ crystallization induced by

second-layer nucleation. ACS Nano 2017;11:865-871. 